\title{
Cases of the Reincarnation Type with Memories from the Intermission Between Lives
}

\author{
Poonam Sharma, B.A. \\ Jim B. Tucker, M.D. \\ University of Virginia
}

\begin{abstract}
For the last 40 years, researchers have collected cases of children who claim to remember previous lives. In a minority of these cases, the subjects also claim to remember events that took place during the intermission between the end of their previous life and their birth in the current life. Subjects in these cases tend to make more verified statements about the previous life they claim to remember than do other subjects of reincarnationtype cases, and they tend to recall more names from that life. Analysis of reports from 35 Burmese subjects indicates that the intermission memories can be broken down into three parts: a transitional stage, a stable stage in a particular location, and a return stage involving choosing parents or conception. A comparison of these reports to reports of near-death experiences (NDEs) indicates that they show features similar to the transcendental component of Western NDEs and have significant areas of overlap with Asian NDEs.
\end{abstract}

KEY WORDS: reincarnation, near-death experiences.

At the University of Virginia Division of Personality Studies, researchers have for the last 40 years collected cases of children who claim to remember previous lives (Stevenson, 2001). These cases of the reincarnation type (CORT) typically involve children who report

Poonam Sharma, B.A., is a medical student, and Jim B. Tucker, M.D., is Assistant Professor of Psychiatric Medicine, at the University of Virginia School of Medicine. This study was supported in part by a grant from the Society for Psychical Research. Reprint requests should be addressed to Dr. Tucker at the Division of Personality Studies, University of Virginia Health System. P.O. Box 800152, Charlottesville, VA 22908-0152; e-mail: jbt8n@virginia.edu. 
memories of a recent life at a very early age, usually beginning around the age of 2 or 3 years and ending by the age of 6 or 7 . The memories they report sometimes involve the life of a family member or an acquaintance. In other cases, however, children have described the life of a stranger in another location, and people have then verified that an individual died whose life matched the description given by the child. We refer to that individual as the previous personality.

These cases often include other features in addition to the child's statements about a previous life. A number of children have been born with birthmarks or birth defects that corresponded to wounds, usually fatal ones, suffered by the previous personality, and many of the children show behaviors that seem related to the previous life, such as phobias related to the mode of death or repetitious play mimicking the previous personality's occupation.

In a minority of the cases, the children have reported memories of events that took place between the death of the previous individual and their own birth. These cases of the reincarnation type with intermission memories, which we will refer to as CORT-I, are the focus of this paper.

\section{Statistical Analysis of CORT-I}

More than 2500 CORT from around the world are now registered in our files, and 1200 of them have been entered into a computerized database using SPSS for Windows, Version 12.0. In 276 of these, the subject has claimed to remember not only a past life, but also a time between lives, an intermission memory. These intermission memories are coded into four categories: claimed memories of the previous personality's funeral, memories of other terrestrial events, memories of an existence in another realm, and memories of conception or of being reborn.

Analyses were performed on the entire collection of CORT that have been entered into the computerized database with the exclusion of cases from Burma (also known as Myanmar). Coding of the Burmese CORT into the database is incomplete, with some cases being chosen because of the presence of intermission memories, so they are not a representative sample useful for statistical analysis. The countries in which the coding has been completed include Eastern countries such as India, Sri Lanka, Turkey, and Lebanon, along with the United States and Canada, and using cases from all countries except for Burma resulted in 1107 cases, with 217 of them having intermission memories. For the analysis, the number of cases varied for each item 


\section{Table I}

\section{Memory of Subjects and Strength of Cases in CORT-I Versus CORT}

\begin{tabular}{lcccrr}
\hline & CORT-I & CORT & $t$ & $d f$ & \multicolumn{1}{c}{$p$} \\
\hline Number of verified statements & 9.07 & 4.92 & -5.04 & 776 & $<.001$ \\
Names from previous life & 3.48 & 2.89 & -2.34 & 670 & .020 \\
Number of lives recalled & 1.37 & 1.02 & -3.15 & 141 & .002 \\
SOCS score & 15.7 & 9.7 & -8.23 & 1054 & $<.001$ \\
\hline
\end{tabular}

based on the number of cases with the relevant data recorded. We used chi-square tests and independent t-tests to analyze these data, with a significance level of $p=.05$.

Table 1 compares CORT-I subjects with other CORT subjects on items related to past-life memories. CORT-I subjects made significantly more statements about the previous life that were verified to be accurate than their CORT counterparts. They remembered more names from the previous life, in addition to the name of the previous personality. They also tended to recall a larger number of past lives. Based on Pearson chi-square tests, CORT-I subjects were significantly more likely to state the name or nickname of the previous personality $\left(\chi^{2}=9.549, \mathrm{df}=1, \mathrm{p}=.049\right)$ and to claim to remember the mode of death $\left(\chi^{2}=5.388, \mathrm{df}=1, \mathrm{p}=.020\right)$. While our database does not indicate specifically whether the names the subjects gave were accurate, the statements by the CORT-I subjects about the mode of death were much more likely to be independently verified than those of other subjects ( $\chi^{2}=23.229, \mathrm{df}=1, \mathrm{p}<.001$ ), and in those cases, 84 percent of their reports of the death were either accurate in most details ( 74 percent) or totally accurate (10 percent).

The CORT-I were also stronger than other CORT based on a Strength-of-Case Scale (SOCS) that assigns points according to four categories of factors: (1) birthmarks or birth defects on the subject that correspond to wounds on the previous personality, (2) verified statements by the subject about the previous life, (3) unusual behaviors related to the previous life, and (4) distance between the previous personality and the family of the subject (Tucker, 2000). The mean SOCS score was 15.7 for CORT-I and 9.7 for CORT.

Other features of the cases failed to differentiate CORT-I subjects from CORT subjects. There were no differences in gender of the subjects: 58 percent of the CORT-I subjects were male, as were 63 
Table II

Characteristics of CORT-I Versus CORT Subjects

\begin{tabular}{lccccc}
\hline & CORT-I & CORT & $t$ & $d f$ & $p$ \\
\hline $\begin{array}{l}\text { Age subject first spoke } \\
\quad \text { (in months) }\end{array}$ & 22.69 & 22.54 & -0.16 & 693 & .872 \\
$\begin{array}{l}\text { Age subject first } \\
\text { communicated about }\end{array}$ & 34.07 & 35.84 & 0.60 & 917 & .549 \\
$\begin{array}{l}\text { past life (in months) } \\
\text { Number of birthmarks }\end{array}$ & 0.84 & 0.93 & 0.42 & 1022 & .681 \\
$\begin{array}{l}\text { Number of birth defects } \\
\begin{array}{l}\text { Distance between previous } \\
\text { personality and subject }\end{array}\end{array}$ & 0.60 & 0.23 & -1.16 & 958 & .247 \\
$\quad$ (in kilometers) & 201 & 255 & 0.41 & 460 & .68 \\
\hline
\end{tabular}

percent of other CORT subjects $\left(\chi^{2}=1.959, \mathrm{df}=1, \mathrm{p}=.162\right)$. As shown in Table 2, there were also no significant differences in the age when the children first spoke, the age when they first communicated about a past life, the number of birthmarks, the number of birth defects, and the distance between the previous personality and the subject. The mean distances shown in Table 2 (201 kilometers for cases with intermission memories and 255 kilometers for other cases) are skewed by a small number of extremely-long-distance cases. The median distances were 20 kilometers for cases with intermission memories and 14 kilometers for other cases.

Similarly, as shown in Table 3, chi-square tests indicated no significant differences in the behaviors of CORT-I subjects and other CORT subjects. The one exception was that subjects who reported intermission memories were less likely to express memories of the previous life in their play, but that difference lost statistical significance when the Bonferroni correction was used to correct for multiple simultaneous statistical tests on related data.

Lastly, we analyzed characteristics of the previous personality with chi-square tests, as shown in Table 4 . These included characteristics both of that individual while living and of his or her death, and none of them had a significant effect on whether the subject in the case later reported intermission memories. The database has information on some of these items for only a small numbers of cases, reducing the power of the tests to detect a significant change, but none of the tests for those items came close to approaching significance. 
Table III

Behaviors of CORT-I Versus CORT Subjects

\begin{tabular}{lcccc}
\hline & $N$ & $\chi^{2}$ & $d f$ & $p$ \\
\hline $\begin{array}{l}\text { Unusual maturity } \\
\text { Adult attitude }\end{array}$ & 661 & 1.633 & 3 & .652 \\
$\begin{array}{l}\text { Unusual dietary cravings } \\
\text { related to previous life }\end{array}$ & 658 & 1.626 & 3 & .804 \\
$\begin{array}{l}\text { Unusual desire for alcohol } \\
\quad \text { or tobacco }\end{array}$ & 310 & 7.414 & 3 & .060 \\
$\begin{array}{l}\text { Unusual philias related to } \\
\text { previous life }\end{array}$ & 495 & 3.784 & 3 & .286 \\
$\begin{array}{l}\text { Unusual phobias related to } \\
\text { previous life }\end{array}$ & 808 & 3.408 & 3 & .333 \\
$\begin{array}{l}\text { Memories of previous life } \\
\quad \text { expressed in play }\end{array}$ & 539 & 4.359 & 1 & .037 \\
$\begin{array}{c}\text { Display of emotion during } \\
\text { recall of previous life }\end{array}$ & 659 & 0.191 & 3 & .979 \\
$\begin{array}{c}\text { Personality change during } \\
\text { recall of previous life }\end{array}$ & 577 & 0.793 & 3 & .851 \\
\hline
\end{tabular}

To summarize, these results indicate that only an unusually strong memory, and not any other characteristic of the subject or previous personality, distinguishes CORT-I subjects from other CORT subjects. The CORT-I subjects not only claim to have memories of the intermission between the deaths of the previous personality and their own births, but they also demonstrate more ability to recall a variety of memories from the past life. CORT-I subjects are more likely to state a name for the previous personality, names of other individuals from the previous life, and the mode of death of the previous personality, and they make significantly more statements about the previous life that have been verified to be accurate. Along with the highly significant differences in the SOCS scores, this indicates that these cases warrant serious consideration.

\section{Analysis of Intermission Experiences}

While claimed memories of an intermission experience are rare in some places, they appear to be relatively common in Burma. While the incidence for all of the Burmese cases collected in our files is unknown, 


\section{Table IV \\ Characteristics of the Previous Personality in CORT-I Versus CORT}

\begin{tabular}{|c|c|c|c|c|}
\hline & $N$ & $\chi^{2}$ & $d f$ & $p$ \\
\hline Mode of death & 908 & 15.189 & 9 & .086 \\
\hline Unexpectedness of death & 587 & 4.074 & 4 & .396 \\
\hline $\begin{array}{l}\text { Was previous personality } \\
\text { a meditator? }\end{array}$ & 33 & 2.159 & 4 & .707 \\
\hline $\begin{array}{l}\text { Was previous personality } \\
\text { attached to wealth? }\end{array}$ & 74 & 0.824 & 3 & .844 \\
\hline $\begin{array}{l}\text { Was previous personality } \\
\text { a criminal? }\end{array}$ & 106 & 3.264 & 4 & .659 \\
\hline $\begin{array}{l}\text { Was previous personality } \\
\text { philanthropic/generous? }\end{array}$ & 88 & 3.217 & 4 & .522 \\
\hline $\begin{array}{l}\text { Was previous personality } \\
\text { religiously observant? }\end{array}$ & 145 & 4.418 & 4 & .352 \\
\hline $\begin{array}{l}\text { Was previous personality } \\
\text { saintly? }\end{array}$ & 68 & 3.241 & 4 & .518 \\
\hline $\begin{array}{l}\text { Did previous personality } \\
\text { have unfinished business? }\end{array}$ & 107 & 6.917 & 5 & .227 \\
\hline
\end{tabular}

Ian Stevenson (1983) determined that 52 out of 230 Burmese cases (23 percent) included such reports. In contrast, only 8 percent of the Turkish cases include intermission reports, and only 2 percent of the Lebanese cases have them. These cases were generally investigated more than 20 years ago, and repeat studies would be needed to ascertain if similar reports could be easily found today.

We are currently in the process of coding Burmese CORT to be entered into our database, and given the relatively common intermission reports in them, we decided to analyze those reports in depth as the cases were being coded. Analysis of 35 Burmese CORT-I led to a new scheme for categorizing the intermission experiences that subjects describe.

First, some information about the Burmese culture will allow some perspective on these cases. The prevalent religion is a combination of long-ago imported Buddhism and native animism. Buddhism is the more formal aspect, and it co-exists with a belief in nature spirits (nats) who are thought to cause trouble unless regularly appeased with gifts and displays of respect. While Buddhism predicts changes in fortune over lifetimes according to accumulated karma, the existence 
of nats is invoked to explain more day-to-day misfortunes, and belief in them is widespread (Nash, Obeyesekere, Ames, Ingersoll, Pfanner, Nash, Moerman, Ebihara, and Yalman, 1966; Spiro, 1978). The religious background of Burma may contribute to the increased reports of intermission memories or to the specifics of those reports.

\section{Development of a Composite Temporal Scheme}

Categorizing CORT-I intermission memories is fraught with pitfalls. Accounts of intermission experiences vary, and no two accounts are identical. There are, however, recurring themes, events, and chronologies. Keeping these points in mind, we developed a three-stage temporal scheme to describe the intermission experience and to provide a starting point for further analysis. It describes the typical chronological progression of CORT-I in Burma and highlights the characteristic events and themes of each stage. Not all three stages were experienced by all subjects, and a few subjects described typical characteristics of a particular stage either earlier or later than would be expected by the progression described below. Nevertheless, the described format suited the majority of the cases and serves as a preliminary categorization plan suitable as a stepping-stone for further analysis.

Stage 1. The first stage could be named the "transitional stage." Nine of 35 Burmese intermission subjects recalled characteristics typical of this period. While interview notes rarely document any references by subjects to their emotional state during the intermission period, the events recalled during the transitional stage would often seem uncomfortable or unpleasant to the Western reader. The events and characters described are associated with the previous life. Subjects may see the preparation of the previous personality's body or the funeral or try to contact grieving relatives, only to find they are unable to communicate with the living. One subject said he did not realize he was dead. Several reported being driven away by the weeping of their relatives, an experience that one subject said made him "hot." This stage often ends as the subject is directed by an elder or an old man dressed in white to a place where he or she then stays for the bulk of the intermission experience.

Stage 2. The second stage is characterized by its marked stability compared to the other two stages. Subjects report living in a particular location or having a schedule or duties to which they must attend. Of the 35 cases so far analyzed, 19 had reports typical of the second stage. 
Nine reported staying in a tree, four in a pagoda, and two remained near the place of death. Reports of seeing or interacting with other discarnate personalities are common (seven subjects). Subjects report varying degrees of comfort during this period. One subject reported being abused by other spirits who threw objects at him, and having to walk long distances. Another woman reported not being able to leave the site where she had buried her jewelry in life, but said that she was far more beautiful as a discarnate and had pretty dresses of gold. Another woman, however, who is very poor in her current life, reported that all her needs were taken care of as a discarnate and that her experience was "quite pleasant."

Stage 3. The final stage is one of choosing parents for the next life or of conception. Eighteen of 35 subjects reported characteristics of this stage. Seven reported following the future parents home, apparently on their own initiative, as the parents passed by while performing everyday tasks, such as bathing or returning home from work. Five more reported being directed to the present parents, often by elders or the old man figure referred to in stage 1 . Nine commented on how they gained entrance to the mother's body. This was most often by transforming into a grain of rice or speck of dust in the water and being ingested by the mother. A few went to considerable lengths, having to try repeatedly when either they were rebuffed by guardian spirits or the water was thrown out as dirty.

Preliminary comparison suggests that this scheme is applicable not only to Burmese CORT-I but to CORT-I internationally. Stevenson (1983) reported the case of Bongkuch Promsin from Thailand, who claimed that, after the death of his previous personality, he lived in a tree for seven years (stage 2) and then followed his current father home on a bus (stage 3). Thai monk Chaokhun Rajsuthajarn reported attending the funeral of the previous personality (stage 1) and trying to interact with the guests, only to realize that he was invisible to them (Stevenson, 1983). Purnima Ekanayake of Sri Lanka reported seeing people crying at her funeral (stage 1) and going to the light to come to her new family (stage 3) (Haraldsson, 2000). Sam Taylor (an unpublished American case from our files) reported that God gave him a card with green arrows to come back from heaven (stage 3). While the specific imagery may be culture-specific, preliminary study suggests that the phases seem to be universally applicable. The further study into the details of international CORT-I required to refine this assertion remains to be done. 


\section{Comparison of CORT-I with Near-Death Experiences}

The recurring thematic elements of CORT-I, particularly the visions of other beings and suggestions of being in another realm, bring to mind accounts of near-death experiences (NDEs). This thematic overlap deserves exploration. An initial comparison was undertaken by application of two NDE scales, the Weighted Core Experience Index (WCEI) (Ring, 1980) and the Near-Death Experience Scale (Greyson, 1983), to the composite temporal scheme outlined above. Some differences in accounts should be expected, since NDErs obviously return to life or continue living, while people who report intermission memories claim to recall a period after they had permanently lost a life. Furthermore, the WCEI and the NDE Scale were developed using accounts from very different cultural surroundings than the CORT-I. This being the case, we explored the differences between NDEs (as outlined in the two scales) and CORT-I using an international review of NDEs and studies of NDEs in Burma's neighbors, India and Thailand.

\section{Comparison of Composite CORT-I with the WCEI}

The WCEI consists of 10 items that are weighted differentially to produce a measure of the depth of an NDE, as outlined in Table 5. It starts with "a subjective sense of being dead" experienced by all 35 of the Burmese CORT-I used for analysis (1 point). The second item, "feeling of peace, painlessness, etc.," was described by only two subjects (one saying, "I was quite happy" and the other stating a more ambiguous, "It appeared to be a very pleasant life"). In fact, more common were statements of discomfort, especially around weeping relatives ("the relatives started weeping. ... I could not bear it"; "We felt very hot if any of our relatives wept for us. ... We would have to flee"; "Weeping scares away discarnates").

Nor did any of the Burmese CORT-I analyzed clearly report the next item, "sense of bodily separation." Some reported watching their funeral (apparently not from the perspective of the body), being able to change form, or not having to eat - characteristics suggestive of a lack of bodily sensation or limitations. However, many (10 subjects) described the clothes they were wearing, usually the clothes of the deceased. The mention of clothes, particularly the clothes of the 


\section{Table V}

The WCEI and CORT-I Experiences

\begin{tabular}{lcl}
\hline \multicolumn{1}{c}{ WCEI Item } & Weight & $\begin{array}{c}\text { Commonly Reported by } \\
\text { CORT-I Subjects }\end{array}$ \\
\hline $\begin{array}{l}\text { Subjective sense of being } \\
\text { dead }\end{array}$ & 1 & Yes \\
$\begin{array}{l}\text { Feeling of peace, } \\
\text { painlessness, etc. }\end{array}$ & 2 & No \\
$\begin{array}{l}\text { Sense of bodily separation } \\
\begin{array}{l}\text { Sense of entering a dark } \\
\text { region }\end{array}\end{array}$ & 2 & No (?) \\
$\begin{array}{l}\text { Encountering a } \\
\text { presence/hearing a voice }\end{array}$ & 3 & No \\
$\begin{array}{l}\text { Taking stock of one's life } \\
\text { Seeing, or being enveloped }\end{array} \quad 3$ & Yes \\
$\quad$ in, light & 2 & No \\
Seeing beautiful colors & & No \\
Entering into the light & 1 & No \\
Encountering visible spirits & 4 & No \\
& 3 & Yes \\
\hline
\end{tabular}

deceased, makes this an ambiguous area of overlap. Based on the descriptions of their actions, however, the subjects seemed to remember being separate from their physical bodies even if they did not immediately recognize it or explicitly state it.

The next item, "sense of entering a dark region," was not reported. Many (nine subjects) did report the next item, however, "encountering a presence/hearing a voice" ( 3 points). This presence was referred as an old man in white, and elder, the Authority, Hades, or the Guardian. The presence usually offered guidance through a period of transition. Only one case reported "taking stock of one's life" (chided by "Hades" for not performing a particular religious ceremony) and none reported either "seeing, or being enveloped in, light," "seeing beautiful colors," or "entering into the light." The last feature, "encountering visible spirits" (3 points), was frequently referred to ( 14 cases). The fact that these spirits as well as the subject were referred to as "discarnates" lends further support to a "sense of bodily separation" mentioned earlier.

Many CORT-I accounts include the three features of a subjective sense of being dead, encountering a presence, and encountering visible 


\begin{tabular}{ll}
\hline \multicolumn{1}{c}{ NDE Scale Item } & $\begin{array}{c}\text { Commonly Reported } \\
\text { by CORT-I Subjects }\end{array}$ \\
\hline Cognitive component: & \\
Time distortion & No \\
Acceleration of thoughts & No \\
Life review & No (?) \\
Sudden understanding & No \\
Affective component: & \\
Peace, pleasantness & Rare \\
Joy & No \\
Cosmic unity & No \\
Seeing or feeling surrounded by light & No \\
Paranormal component: & \\
Senses more vivid than usual & No \\
Extrasensory perception & No \\
Precognitive visions & No \\
Out-of-body experience & No (?) \\
Transcendental component: & \\
Entering an unearthly world or dimension & Yes \\
Encountering a mystical being or presence & Yes \\
Seeing deceased or religious spirits & Yes \\
Border or point of no return & No \\
\hline
\end{tabular}

spirits, which would score a 7 on the WCEI, falling into Ring's category of a "moderate experience." Inclusion of "a sense of bodily separation" would push the composite temporal experience into the "deep experience" category. Clearly, this is enough to indicate a similarity between the reports of the two experiences.

\section{Comparison of Composite CORT-I with the NDE Scale}

While comparison to the WCEI establishes areas of overlap between CORT-I and NDEs as a whole, comparison of CORT-I with the NDE Scale highlights the contrasts between the two experiences (Table 6). 
The questionnaire is divided into four categories with four questions each. The categories highlight the cognitive, affective, paranormal, and transcendental aspects of the NDE. This categorization of NDE features allows a clearer picture of how NDEs and intermission memories in CORT-I differ.

None of the CORT-I subjects claimed a clear cognitive change as a discarnate. Far from talking about an increase in the speed of time (question 1), subjects who referred to time reported definite time periods with no reference to a change in the rate of time, such as, "I was a discarnate for 7 days." No subjects reported a change in the speed of thoughts (question 2). Two subjects made statements that, if stretched, may indicate past scenes coming back to them (question 3). One said that Hades chided her for not performing a religious ceremony. Another said that spirits were shown places where they had lived and asked if they yearned for those earthly places. None of the 35 cases claimed to experience a universal understanding (question 4).

Comparison of CORT-I with the affective component of the questionnaire reveals even more differences. Only 2 of the 35 cases, as discussed before, suggested a feeling of peace or pleasantness (question 5 ), while several reported uncomfortable experiences. None suggested a feeling of joy (question 6) or a feeling of harmony or unity with the universe (question 7), or saw or felt surrounded by a brilliant light (question 8).

Similarly, comparison with the four paranormal questions brings out few similarities. No CORT-I subjects reported a heightening of senses (question 9), although one reported an ability to see auras during his time as a discarnate. None reported a sense of awareness of things going on elsewhere (question 10). None reported scenes from the future (question 11). And none clearly stated feeling separated from their body (question 12).

The transcendental aspect of the questionnaire displays dramatically different results. Reports of another realm were common (question 13). As discussed earlier, many encountered a mystical being or presence, such as an old man in white, Hades, or the Guardian (question 14). Many (14 subjects) also referred to deceased spirits they saw during their period as a discarnate (question 15). None reported coming to a border or point of no return, perhaps not surprisingly since they claimed to have passed on to death and not returned to life.

To summarize, both NDEs as defined by the WCEI and CORT-I include a sense of being dead, encountering a presence (often referred to as an old man in white in CORT-I) and encountering visible spirits (other discarnates or deceased relatives). Comparison with the NDE 
Scale highlights the relative lack of reports of cognitive, affective, or paranormal aspects to the death experience described by CORT-I subjects, and the sizable overlap in transcendental aspects of the two experiences.

\section{Comparison of Composite CORT-I with International NDEs}

Both the WCEI and NDE Scale were developed from American accounts of NDEs. The few comparisons of NDEs from Hindu and Buddhist cultures suggest there are definite cross-cultural variations (Murphy, 2001; Pasricha and Stevenson, 1986; Pasricha, 1995). Thus, the discrepancies between NDEs and CORT-I described so far may be due to the application of Western standards to a Burmese sample. Ideally, CORT-I studied in Burma with would be compared to NDE accounts of Burmese subjects, but since no study of Burmese NDEs has yet been undertaken, we will use the few works on international NDEs as a serviceable proxy to review the remaining differences between NDE accounts and the experiences recalled by CORT-I subjects.

Allan Kellehear (1993) reviewed published reports of NDEs from China, India, Western New Britain, Guam, Native North America, Aboriginal Australia, and Maori New Zealand, with the number of cases in some places being very small. After reviewing several elements of NDEs widespread among Western cases (tunnel experience, out-ofbody experience [OBE], life review, encounters with other beings, and another world) he found the major cross-cultural features to be encountering other beings and other worlds, two common characteristics of CORT-I. The tunnel experience, missing from CORT-I, was not reported in any of the nonWestern NDEs that Kellehear reviewed, though experiences of darkness were often present. Other features present in several but not all cultures' NDEs were also present in CORT-I. Out-of-body experiences, which were at least suggested in CORT-I, were reported from India, Guam, Native North America, and Maori New Zealand, but not from China or Aboriginal Australia and probably not from Western New Britain. The life review present in one CORT-I was reported in China, India, and probably Western New Britain, but not from Guam, Native North America, Aboriginal Australia, or Maori New Zealand. The only features that Kellehear found in NDEs from all cultures - seeing other beings and other realms - are readily apparent in CORT-I as well, and CORT-I may resemble Western NDEs as much as do the NDEs of some other cultures. 


\section{Indian NDEs and CORT-I}

Similar to Kellehear's results, Satwant Pasricha (1995) found both similarities and differences between cross-cultural accounts of NDEs. Both Indian and American subjects reported meeting dead relatives and acquaintances, seeing beings of light or religious figures, being revived through the thoughts of loved living persons, being sent back from the other realm by a deceased loved one, and seeing their own physical body while ostensibly dead. The last two characteristics were not found among North Indian cases and so may not be universal characteristics. Being sent back by the thoughts or actions of loved ones is not a criterion applicable to the study of CORT-I, since CORT-I subjects claim not to have been sent back. The remaining two characteristics, meeting deceased relatives and seeing beings of light or religious figures, seem to correlate with CORT-I accounts.

Pasricha also reported six characteristics of Indian NDEs that are not seen in American NDEs. Four involve returning to life and are therefore not relevant to CORT-I, but the other two are that the subject was "taken to other realms by messengers or some one" and then was "passed on to the man with a book" containing a list of deeds or mistakes (Pasricha, 1995, p. 86). While the Burmese CORT-I subjects did not report the latter, their reports of being directed to a new place by an elder or an old man dressed in white at the end of the transitional phase sound very similar to the former.

\section{Thai NDEs and CORT-I}

Both India and Thailand border Burma, but Thailand appears to have a more similar religious climate. While Burmese supernaturalism has not permeated Thailand, Theravada Buddhism is the religion of the overwhelming majority in both countries.

Todd Murphy (2001) gathered a collection of ten NDE cases from Thai popular literature. The reported imagery seemed to be very much affected by the culture, though Murphy argued that it should actually be considered a reflection of the individuals' expectations of what death would be like rather than a reflection of their culture itself. The themes he reported were Yamatoots (servants of the God of death, Yama), the importance of merit accumulated during life, and the presence of cases of mistaken identity.

The appearance of the Yamatoots seemed to act in concert with OBEs to convey to the subjects that they were deceased and separate 
from their bodies. Murphy noted that while OBEs might be found in the NDEs of all cultures, some may not recognize them as a herald of death, and thus, anthropomorphic harbingers of death such as Yamatoots might be necessary to reinforce the awareness of death. No correspondence to the Yamatoots is seen in the Burmese CORT-I, but they are seen frequently in Indian NDEs (Pasricha and Stevenson, 1986). The Burmese reports are more suggestive of out-of-body experiences like those of a typical Western NDE, even if the subjects are not aware of separating from their bodies. Rather than being explicitly told that they are dead, Burmese subjects seemed to figure it out on their own, although a few subjects reported that it took them up to 7 days to realize it.

The second Thai characteristic, postmortem assessment of karma by Yama, seems to be similar to a life review in Western NDEs, with some significant differences. The Thai version involves either explicit physical suffering as punishment for the accumulation of bad karma (for example, being forced to go thirsty if the subject never offered drinks to monks) or implied suffering in future lives (for example, rebirth as a bird). Also, Murphy reported that some cases seemed to assess the balance of good and bad done during life rather than the moment-by-moment life review reported in Western NDEs. This type of life review bears more similarity to Pasricha's account of Indian NDEs, where messengers pass the subject on to a man with a book, rather than the Western panoramic life review. It is hard to determine from the one reported case of a life review in the Burmese CORT-I studied which model would be a more appropriate description for CORT-I generally. In that case, however, the subject reported being chided by Hades for not performing a particular religious ceremony. The reference to one specific lack and the explicit mention of a direct reprimand suggest that in that case, the life review was more similar to Asian NDEs in this regard.

The third characteristic Murphy reported, cases of mistaken identity, are also found in Indian cases but not in Western NDEs. No reports of mistaken identity have been found in Burmese CORT-I, perhaps unsurprisingly, since the subjects all died rather than getting "sent back" in favor of the correct person. The universal features pointed out by Kellehear and Pasricha and present in CORT-I are also present in the Thai NDEs. For example, dead friends and relatives were seen in four of Murphy's 10 cases.

One of the most striking differences between Western NDEs and Burmese CORT-I so far described is the significant lack of positive 
affect so frequently described in the Western near-death experiences. This positive affect also seems to be missing from Thai NDEs. Murphy reported that while some subjects claimed to see heaven and feel "pleasantness, comfort, a sense of beauty, and happiness," none reported the "ineffable" experience so often relayed by NDErs in the West. And just as common, Murphy reported, were reports of negative affect. Five of his subjects reported seeing the tortures of hell, and the reports of seeing heaven or hell are similar, in theme if not in specifics, to reports of other realms described by CORT-I subjects.

Lastly, Murphy reported an absence of the light and tunnels in Thai NDEs. While both are common NDE images in the West, they seem to be missing from both Thai NDEs and Burmese CORT-I.

\section{Conclusion}

Cases of the reincarnation type with intermission memories are a striking phenomenon, but since the memories are usually unverifiable, they have not been a significant focus of research up to this point. The current analysis, however, suggests that they should be carefully considered. Since the children who report such memories tend to make more verified statements about the previous life they claim to remember than do other subjects, and tend to recall more names from that life, their reports of events from the intermission period seem to be part of a pattern of a stronger memory for items preceding their current lives.

An examination of the content of the intermission memories indicates significant overlap with reports of NDEs. For the Western NDEs, this is particularly true for the transcendental aspects of those reports. For NDE reports from other parts of the world, the universal features of NDEs are seen in the intermission descriptions, and the Burmese intermission reports are similar, though not identical, to NDE reports from neighboring India and Thailand. While the differences in the reports should not be glossed over, the similarities indicate that the intermission reports by children claiming to remember previous lives may need to be considered as part of the same overall phenomenon reports of the afterlife - that encompasses NDEs.

If the intermission reports are part of an overall phenomenon, then what do they reveal about that phenomenon? As with NDEs, the reports show individual differences and contain features that seem to be at least culturally connected, if not culturally driven. More universal themes, 
however, appear to underlie those features. They include a recognition of dying, either through an OBE or through a visit from a harbinger of death. There is often then an experience with spirits or a visit to, or view of, another realm. This is frequently followed by memories of returning to life, either in a new body in the intermission cases or by reaching a point of no return and turning back in the case of NDEs.

While the latter two themes might be thought of as attempts to deny the reality of death, the first can hardly be so. In fact, the argument that NDErs create their fantasies of an afterlife as a defense against the confrontation with death is weakened by the similar intermission reports. The subjects in these cases are young children who have not been close to death and in fact would not be thought able to comprehend the concept of death; yet their reports bear many similarities to NDE reports, thus posing a problem for psychological explanations offered for NDEs. Likewise, the neurophysiological explanations that have been offered cannot explain the similar reports from healthy, young children.

More work needs to be done with the intermission memory cases; in particular, reports from various cultures need to be closely analyzed. Further exploration of these cases may also provide additional insights into phenomena such as NDEs.

\section{References}

Greyson, B. (1983). The near death experience scale: Construction, reliability, and validity. Journal of Nervous and Mental Disease, 171, 369-375.

Haraldsson, E. (2000). Birthmarks and claims of previous-life memories: I. The case of Purnima Ekanayake. Journal of the Society for Psychical Research, 64, 16-25.

Kellehear, A. (1993). Culture, biology, and the near death experience: A reappraisal. Journal of Nervous and Mental Disease, 181, 148-156.

Murphy, T. (2001). Near-death experiences in Thailand. Journal of Near-Death Studies, 19, 161-178.

Nash, M., Obeyesekere, G., Ames, M. M., Ingersoll, J., Pfanner, D. E., Nash, J. C., Moerman, M., Ebihara, M., and Yalman, N. (1966). Anthropological studies in Theravada Buddhism. New Havén, CT: Yale University.

Pasricha, S. (1995). Near death experiences in south India: A systematic survey. Journal of Scientific Exploration, 9, 79-88.

Pasricha, S., and Stevenson, I. (1986). Near death experiences in India: A preliminary report. Journal of Nervous and Mental Disease, 174, 165-170.

Ring, K. (1980). Life at death: A scientific investigation of the near-death experience. New York, NY: Coward, McCann and Geoghegan.

Spiro, M. E. (1978). Burmese supernaturalism (expanded ed.). Philadelphia, PA: Institute for the Study of Human Issues. 
Stevenson, I. (1983). Cases of the reincarnation type, Vol. IV: Twelve cases in Thailand and Burma. Charlottesville, VA: University Press of Virginia.

Stevenson, I. (2001). Children who remember previous lives: a question of reincarnation (rev. ed.). Jefferson, NC: McFarland.

Tucker, J. B. (2000). A scale to measure the strength of children's claims of previous lives: Methodology and initial findings. Journal of Scientific Exploration, 14, 571-581. 